\title{
About Unusual Properties of Kinematical Magneto-Electric Systems Possessing Spherical Geometry
}

\author{
Vladimir A. Leus ${ }^{1}$ \\ ${ }^{1}$ Department of Electrical Engineering and Electronics, University of Liverpool, Liverpool L69 3GJ, UK \\ Correspondence: Vladimir A. Leus, Department of Electrical Engineering and Electronics, University of \\ Liverpool, Liverpool L69 3GJ, UK. E-mail: V.Leus@liverpool.ac.uk
}

\author{
Received: October 18, 2015 Accepted: October 30, 2015 Online Published: November 19, 2015 \\ doi:10.5539/apr.v7n6p95 \\ URL: http://dx.doi.org/10.5539/apr.v7n6p95
}

\begin{abstract}
It is common knowledge that properties of any system cannot be reduced to the sum of properties of its elements as relations between elements play an important role in the system structure and therefore in the system behavior. In the given paper the systems constructed from rather simple well-known elements, which spatial interposition is characterized by spherical symmetry, are examined. Each element is a source of a field that is easy calculated. However the result of superposition of these fields is beyond the scope of classical notions.
\end{abstract}

Keywords: magneto-electric systems, spherical symmetry, superposition of kinematically begotten fields

\section{Introduction}

There exist in the nature two completely different mechanisms of field's transmission (Leus, Taylor, 2012; Leus et al., 2013a, 2013b). In an electromagnetic wave vectors $\mathbf{E}$ and $\mathbf{B}$ do not move at all, but synchronously vary their values in space. On the contrary, magnetic field of a moving permanent magnet moves in space (Zajev-Dokuchajev effect), and electric field of a moving charge moves in space (Rowland-Eihenvald effect) together with their carriers like a rigid body (Leus, 2013). These both effects are of purely kinematical origin and they have nothing to do with electro-dynamic signals propagating in space with the light speed (Leus \& Smith B., 2014).

Consider the following situation. There is a narrow gap across the cylindrical bar-magnet where fringing effects are to be neglected and, therefore, magnetic field is very nearly uniform inside with vector $\mathbf{B}$ directed up vertically (Figure 1). If the bar-magnet (both parts) rotates about its central axis with an angle velocity $\omega$ (for example, counterclockwise if viewing from the top), then an electric field $\mathbf{E}=\mathbf{B} \times \mathbf{v}$ is induced inside the gap, due to the Zajev-Dokuchajev magneto-kinematical effect (Leus \& Zatolokin, 2006), which is a natural consequence of "drugging" of the field by a moving carrier.

From the times of Faraday the physicists are divided into two opposite parties: 'fixists', who believe that magnetic field remains steady when its carrier - permanent magnet rotates, and 'mobilists', who are confident the field does rotate, i.e. "the lines of force sit fixed on a steel magnet, like bristles on a brush" as expressed by a German physicist R.W. Pohl (Pohl, 1960). That is why this point of view is termed 'the bristle theory'. Recently in the series of delicate experiments the validity of the latter has been irrefutably proved (Leus \& Taylor, 2011; Taylor \& Leus, 2012). "The moving field theory or bristle theory is not only simpler, but also more logical, more natural, more plausible, more consistent and more economical. Furthermore, not contradicted by any theoretical or experimental evidence, it helps students visualize the relevant electromagnetic processes more vividly than the fixed field theory" (Rajaraman, 2008). Therefore magnetic flux moves synchronously with the magnet and consequently the magnet's material cannot be polarized.

In the rectangular coordinates $(x, y, z)$ the magnetic induction $\mathbf{B}$ has components $(0,0, B)$, the linear velocity $\mathbf{v}$ has components $(-\omega x, \omega y, 0)$ and the electric tension $\mathbf{E}$ has components $(-B \omega x,-B \omega y, 0)$. Thus we have a vector field converging to the axis of rotation and progressively diminishing to the zero-value in the very centre. We deduce from this that $\operatorname{curl} \mathbf{E} \equiv \mathbf{0}$ and $\operatorname{div} \mathbf{E}=-B \omega-B \omega+0=-2 B \omega \neq 0$ without any charges everywhere in the gap, i.e. in this instance the stationary electric field cannot satisfy the Maxwell's equations. 


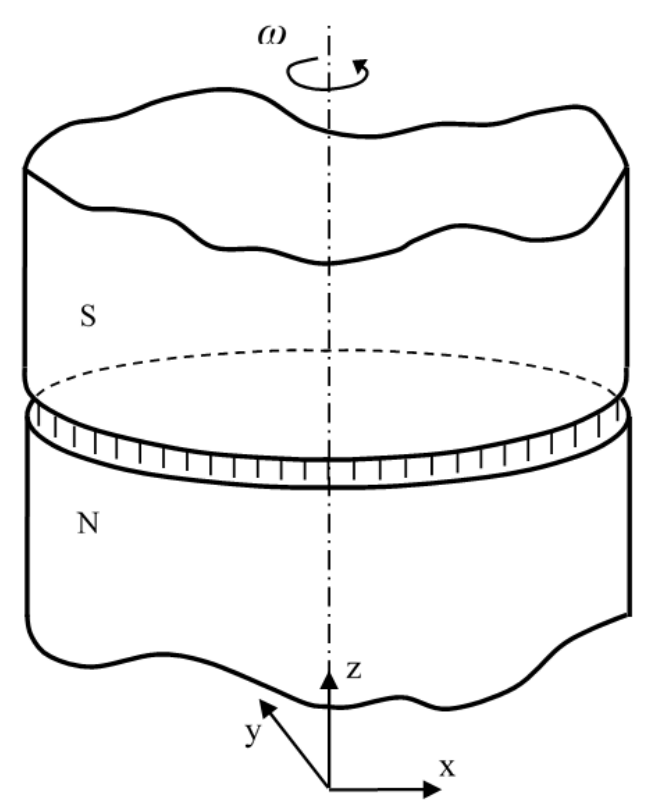

Figure 1. Electric field induced in the narrow pole gap

In the book 'Classical Electricity and Magnetism' (Panofsky \& Phillips, 1975, p. 338) you can read the following would-be comment to the above instance: "Note, however, that the effective electric field in the rotating frame is given by $\mathbf{E}_{r}=\omega B \mathbf{r}$ for low velocities; this expression has a non-vanishing divergence, and thus a volume charge is developed". The authors are so much right as wrong in their assertion: electricity of kinematical origin, indeed, is developed, but it does exist in the laboratory's non-rotating frame. As for " $a$ volume charge", it is putative rather than real, and it would be much more consistent to say: " $\mathbf{E}_{r}$ has a non-vanishing divergence as if a volume charge were developed".

Let us consider one more example of a kinematically begotten field, this time due to the conjugate Rowland-Eihenvald effect. A sphere made of a conductive material is charged with a constant superficial density so that the full charge $q$ is uniformly distributed over all the surface of the sphere. In this case the electrostatic field in air outside the sphere coincides with the field of a point charge $q$ focused in the centre of the sphere:

$$
\mathbf{E}=\frac{q \boldsymbol{r}}{4 \pi \varepsilon_{0} r^{3}} .
$$

At any point $A$ electric field vector $\mathbf{E}$ is collinear with the radius-vector $\boldsymbol{r}$ of this point having Cartesian coordinates $x, y, z$ (Figure 2).

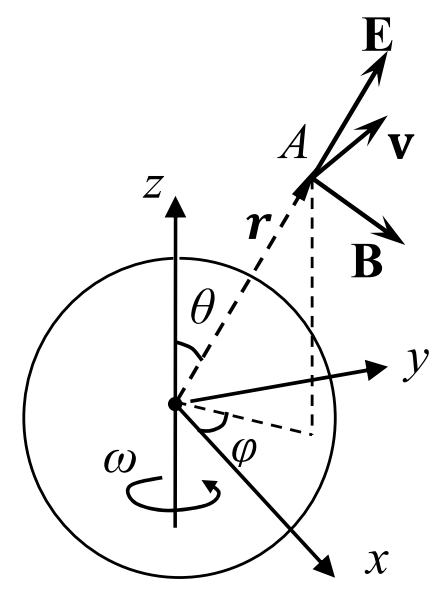

Figure 2. Magnetic field induced by rotating charged sphere

With an accuracy to the constant factor $q / 4 \pi \varepsilon_{0}$ electric vector $\mathbf{E}_{x, y, z}$ has the components 


$$
E_{x}=\frac{x}{r^{3}}, \quad E_{y}=\frac{y}{r^{3}}, \quad E_{z}=\frac{z}{r^{3}},
$$

where $r$ is the length of radius-vector: $r=\sqrt{x^{2}+y^{2}+z^{2}}=(\ldots)^{1 / 2}$. Instantaneous velocity $\mathbf{v}$ at a point $(x, y, z)$ has the absolute value $v=\omega r \sin \theta=\omega \sqrt{x^{2}+y^{2}}=\omega \rho$, and the components $\mathrm{v}_{x}=-v y / \rho=-\omega y$, $\mathrm{v}_{y}=v x / \rho=\omega x, \mathrm{v}_{z}=0$. Lorentz transform gives the following expression for magnetic vector: $\mathbf{B}=\gamma / \mathrm{c}^{2}(\mathbf{v} \times \mathbf{E})$, where $\mathrm{c}$ is light speed, and the relativistic gamma-factor

$$
\gamma=1 / \sqrt{1-v^{2} / c^{2}}=\left[1-\frac{\omega^{2}}{c^{2}}\left(x^{2}+y^{2}\right)\right]^{-1 / 2}=\left[1-b^{2}\left(x^{2}+y^{2}\right)\right]^{-1 / 2}=[. .]^{-1 / 2} .
$$

So, its components are

$$
B_{x}=\frac{\omega}{c^{2}} \cdot \frac{\gamma}{r^{3}} x z, B_{y}=\frac{\omega}{c^{2}} \cdot \frac{\gamma}{r^{3}} y z, \quad B_{z}=-\frac{\omega}{c^{2}} \cdot \frac{\gamma}{r^{3}}\left(x^{2}+y^{2}\right),
$$

Denote $f(x, y, z)=\gamma / r^{3}=[. .]^{-1 / 2} \cdot(\ldots)^{-3 / 2}$ and partial derivatives will be

$$
\begin{gathered}
\frac{\partial f}{\partial x}=b^{2} x[. .]^{-3 / 2} \cdot(\ldots)^{-3 / 2}-3 x[. .]^{-1 / 2} \cdot(\ldots)^{-5 / 2}, \\
\frac{\partial f}{\partial y}=b^{2} y[. .]^{-3 / 2} \cdot(\ldots)^{-3 / 2}-3 y[. .]^{-1 / 2} \cdot(\ldots)^{-5 / 2} \\
\frac{\partial f}{\partial z}=-3 z[. .]^{-1 / 2} \cdot(\ldots)^{-5 / 2} .
\end{gathered}
$$

Then, using the differentiation rule, we can write:

$$
\frac{\partial B_{x}}{\partial x}=\frac{\omega}{c^{2}}\left(z f+x z \frac{\partial f}{\partial x}\right), \quad \frac{\partial B_{y}}{\partial y}=\frac{\omega}{c^{2}}\left(z f+y z \frac{\partial f}{\partial y}\right), \quad \frac{\partial B_{z}}{\partial z}=-\frac{\omega}{c^{2}}\left(x^{2}+y^{2}\right) \frac{\partial f}{\partial z} .
$$

Substitution of the above expressions for derivatives $\frac{\partial f}{\partial x}, \frac{\partial f}{\partial y}, \frac{\partial f}{\partial z}$ allows us to calculate the divergence for the magnetic field of kinematical origin. Rather cumbersome manipulations having omitted, the final result may be present as follows:

$$
\operatorname{div} \mathbf{B}=\frac{\omega Z}{c^{2}}[. .]^{-1 / 2} \cdot(\ldots)^{-3 / 2}\left[2+\frac{b^{2}\left(x^{2}+y^{2}\right)}{1-b^{2}\left(x^{2}+y^{2}\right)}\right]=\frac{\omega z}{c^{2}} \cdot \frac{\gamma}{r^{3}}\left(2+\frac{\omega^{2} \rho^{2} / c^{2}}{1-\omega^{2} \rho^{2} / c^{2}}\right) .
$$

So, divergence of the magnetic field of kinematical origin in this case is essentially non zero. Naturally, this approximation is valid only for distances $\rho<c / \omega$, because a linear speed $v=\omega \rho$ cannot exceed the light speed $c$. However, even for $\gamma \cong 1$ the divergence remains different from zero. Taking account of Fitzgerald-Lorentz contraction leads to the general-relativistic bending of space.

In this respect it seems relevant to remind a scientific paper "A Question in General Relativity" published three quarters of century ago under initiative by J.R. Oppenheimer. 'Consider two concentric spheres with equal and opposite total charges uniformly distributed over their surfaces. When the spheres are at rest, the electric and magnetic fields outside the spheres vanish. When the spheres are in uniform rotation about an axis through their centre, the electric field outside vanishes, while the magnetic field does not" (Schiff, 1939). In itself, this is an example of spherical capacitor containing all the electric field inside them. However, it is necessary to stipulate the obligatory opposite direction for rotation both spheres. Here we have a curious but not surprising feature because of billions and billions similar examples before our eyes: magnetic field existing around a neutral wire with direct current. "Getting ahead of the story, it is interesting to underline that, if the field of a moving charge is subject to dilatation, any wire with current (conductivity electrons are moving with velocity $\mathbf{v}$ ) must seem to be negatively charged. And, if the same wire is moving with velocity $(-\mathbf{v})$, it must seem positively charged " (Leus, 2014). An electric field outside the file of charged particles was examined depending on the speed $v$ of them. At a distance $h$ from the wire of length $2 s$ the difference between values of electric strengths provided by moving and stationary charge distributions is

$$
E_{v}-E_{0}=\left(\sigma h / 4 \pi \varepsilon_{0} s^{2}\right) \cdot(v / c)^{2},
$$

where $\sigma$ denotes the linear density of charge. This so called effect of the second order had been, possibly, observed in the well-known experiment by Edwards and Lemon, who used a superconductive ring with direct current. It is obvious that for a direct current in ordinary wire the difference $E_{v}-E_{0}$ is not perceptible. Another 
matter when an electron beam instead of wire is dealt. In this case $v / c \approx 1$ and the effect should be thoroughly appreciable (Leus, 2014).

Could we say that the Maxwell's equations are incorrect? On no account! It would be best to speak about a validity region. As it is thought, the boundary lies somewhere between electrostatics-electrodynamics on the one hand, and magneto-electro-kinematics on the other hand. Here bellow we consider some more complicated cases of unusual magneto-electro-kinematic phenomenon arising due to superposition principle. Please, don't forget that these 'true oddities' pertain to a newfound realm in electromagnetism.

In sections 2-4 detailed description of a sophisticated system is present, starting from one magnetic rotator and gradually reaching the final version of many magnetic rotators situating over sphere. In section 5 a reciprocally symmetric system of many electric rotators is briefly considered using niceties of the previous magnetic example. All intermediate geometrical proves are transferred into appendix.

\section{A Solid Magnetized Ball}

"Although physicist often use the term 'sphere' to mean the solid ball, mathematicians definitely do not" (Weisstein, 1999, p. 94). Although the very gist of the paper is particularly physical, we shall use the term 'ball' to mean the round body but the term 'sphere' to mean the surface of such a body. A solid magnetized ball of radius $a$ is the basic unit from which our spherical magneto-system is made up (the main composing element for our spherical magneto-system). A ball with a uniform permanent magnetization $\mathbf{M}$ parallel to the $z$ axis is embedded in a non-permeable medium --- vacuum to be exact. Its field is a pure field of a magnetic dipole (no an azimuthal component) with dipole moment $\mathbf{m}=4 / 3 \pi a^{3} \mathbf{M}$. "For the sphere with uniform magnetization, the fields are not only dipole in character asymptotically, but also close to the sphere. For this special geometry (and this only) there are no higher multipoles" (Jackson, 1975, p. 195). The external magnetic field B is described by the following equations

$$
B_{r}=\frac{2 \mu_{0} m \cos \vartheta}{r^{3}}, B_{\vartheta}=\frac{\mu_{0} m \sin \vartheta}{r^{3}}, B_{\varphi}=0,
$$

where $\mathbf{r}$ is radius vector (with magnitude $r$ ) of any point, $\vartheta$ is a polar angle, $\varphi$ is an azimuth (Figure 3). Here we explicitly introduced the permeability of vacuum $\mu_{0}$ because of use the SI units.

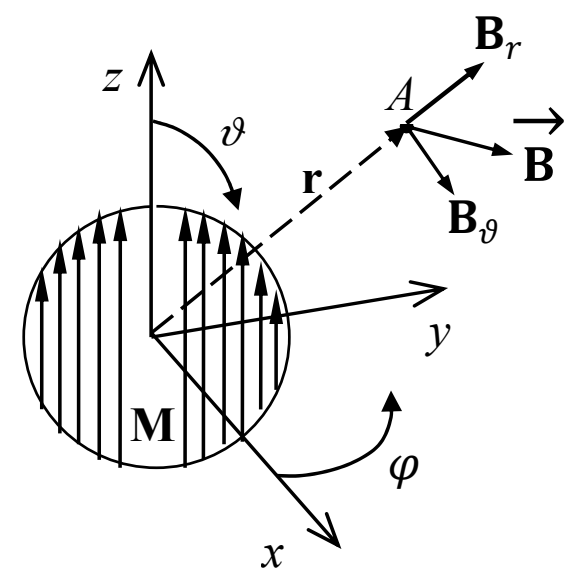

Figure 3. The uniformly magnetized sphere

When a magnetized ball rotates about $z$ axis with an angular speed $\omega$, we have a magnetic rotator (m-rotator). In spherical coordinates $(r, \vartheta, \varphi)$ linear velocity $\mathbf{v}$ providing from rotation has just one nonzero azimuthal component $v_{\varphi}=\omega r \sin \vartheta$. Let us consider electric field $\mathbf{E}=\mathbf{B} \times \mathbf{v}$ induced outside the ball due to the Zajev-Dokuchajev effect. Despite, $\vartheta, \varphi$ are curvilinear coordinates vector product has to be calculated by the same formulae that are valid for Cartesian coordinates because spherical coordinates are orthogonal ones. This gives:

$$
E_{r}=\frac{\mu_{0} \omega m \sin ^{2} \vartheta}{r^{2}}, E_{\vartheta}=-\frac{\mu_{0} \omega m \sin 2 \vartheta}{r^{2}}, E_{\varphi}=0 ; \quad \mathbf{E}=E_{r} \mathbf{u}_{r}+E_{\vartheta} \mathbf{u}_{\vartheta}+E_{\varphi} \mathbf{u}_{\varphi}
$$


where $\mathbf{u}_{r}, \mathbf{u}_{\vartheta}, \mathbf{u}_{\varphi}$ are unit vectors. These equations have been given first in the work of Bogach (Bogach, 1996). It is obvious that the direction of a vector $\mathbf{E}$ is constant along the each radial line i.e. the coordinate line with constant polar angle. Absolute value of a vector $\mathbf{E}$ is

$$
E=\sqrt{{E_{r}{ }^{2}+E_{\vartheta}{ }^{2}}^{2}}=\frac{\mu_{0} \omega m \sin \vartheta}{r^{2}} \sqrt{1+3 \cos ^{2} \vartheta} .
$$

The angle $\propto$ between vector $\mathbf{E}$ and unit radial vector $\mathbf{u}_{r}$ is defined by the equation

$$
\propto=\arccos \left(\frac{\mathbf{E} \cdot \mathbf{u}_{r}}{E}\right)=\arccos \left(\frac{\sin \vartheta}{\sqrt{1+3 \cos ^{2} \vartheta}}\right) .
$$

Here the vector length and inner product $\mathbf{E} \cdot \mathbf{u}_{\mathbf{r}}$ have been calculated by ordinary formulae due to the $r, \vartheta$ are orthonormal coordinates. Thus the tilt to the radius-vector depends on the angle $\vartheta$ only. From this above we get

$$
\cos \propto=\frac{\sin \vartheta}{\sqrt{1+3 \cos ^{2} \vartheta}} ; \sin \propto=\frac{2 \cos \vartheta}{\sqrt{1+3 \cos ^{2} \vartheta}} .
$$

Let us take the square:

$$
\left(1+3 \cos ^{2} \vartheta\right) \cos ^{2} \propto=\sin ^{2} \vartheta ;\left(1+3 \cos ^{2} \vartheta\right) \sin ^{2} \propto=4 \cos ^{2} \vartheta .
$$

With a little manipulation these formulae can be recast to

and then into

$$
4 \cos ^{2} \propto-3 \cos ^{2} \propto \sin ^{2} \vartheta=\sin ^{2} \vartheta ; \sin ^{2} \propto+3 \sin ^{2} \propto \cos ^{2} \vartheta=4 \cos ^{2} \vartheta
$$

where from we have

$$
4 \cos ^{2} \propto=\left(1+3 \cos ^{2} \propto\right) \sin ^{2} \vartheta ; \sin ^{2} \propto=\left(4-3 \sin ^{2} \propto\right) \cos ^{2} \vartheta
$$

$$
\sin ^{2} \vartheta=\frac{4 \cos ^{2} \propto}{1+3 \cos ^{2} \propto} ; \cos ^{2} \vartheta=\frac{\sin ^{2} \propto}{4-3+3 \cos ^{2} \propto}
$$

At last:

$$
\sin \vartheta=\frac{2 \cos \alpha}{\sqrt{1+3 \cos ^{2} \alpha}} ; \cos \vartheta=\frac{\sin \propto}{\sqrt{1+3 \cos ^{2} \alpha}} .
$$

So we get symmetric dependences (3) and (4) between angles $\propto$ and $\vartheta$.

\section{Two Symmetrically Posed Magnetic Rotators}

Let be a sphere of radius $b$, i.e. $b$ - sphere. There are a lot of the similar magnetic rotators of radii $a(a \ll b)$, i.e. $a$-rotators. These magnetic rotators are distributed all over a sphere in such a manner that their centres are located on the spherical surface and the axis of every rotator is directed to the centre of the sphere (Figure 4). Let the internal end of the every rotator's axis be south one $(S)$. Let the direction of rotation be counter-clockwise when viewed from outside of the sphere. Our aim is to calculate the external field induced due to the Zajev-Dokuchajev effect.

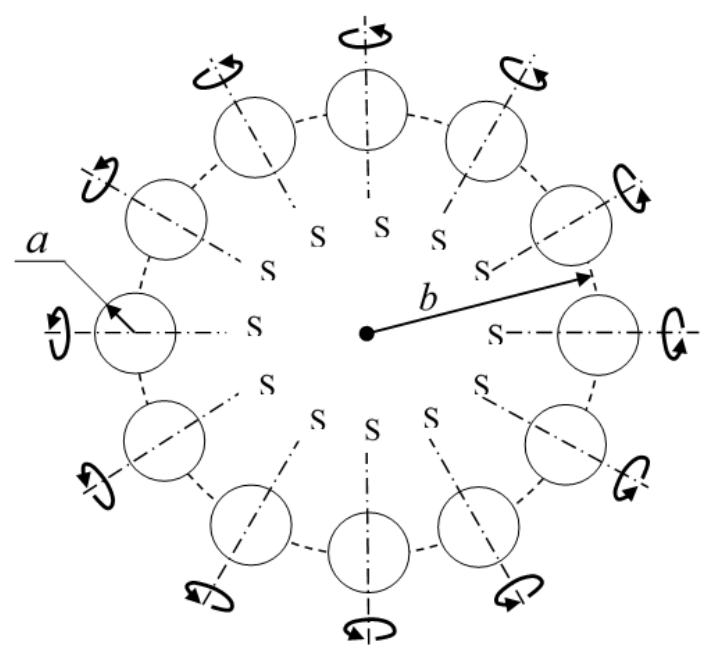

Figure 4. Spherical system of magnetic rotators 
Consider first a simple case of only two $a$-rotators that are located on the $b$-sphere symmetrically to the ray coming from the sphere centre $O$ to a given point of view $P$ (Figure 5). To find the strength of the induced electric field $\mathbf{E}$ at this point, we must compute the vector sum of $\mathbf{E}_{1}$ due to the upper rotator and $\mathbf{E}_{2}$ due to the lower one. Let us use the following geometric statement:

When the distance $R$ between the point $P$ and the centre $O$ is bigger than $2 b$, the orthogonal projection of vector $\mathbf{E}_{1}$ at the direction of radial ray $O P$ holds its sign for all values of positioning angle $\delta$ between 0 and $\pi$.

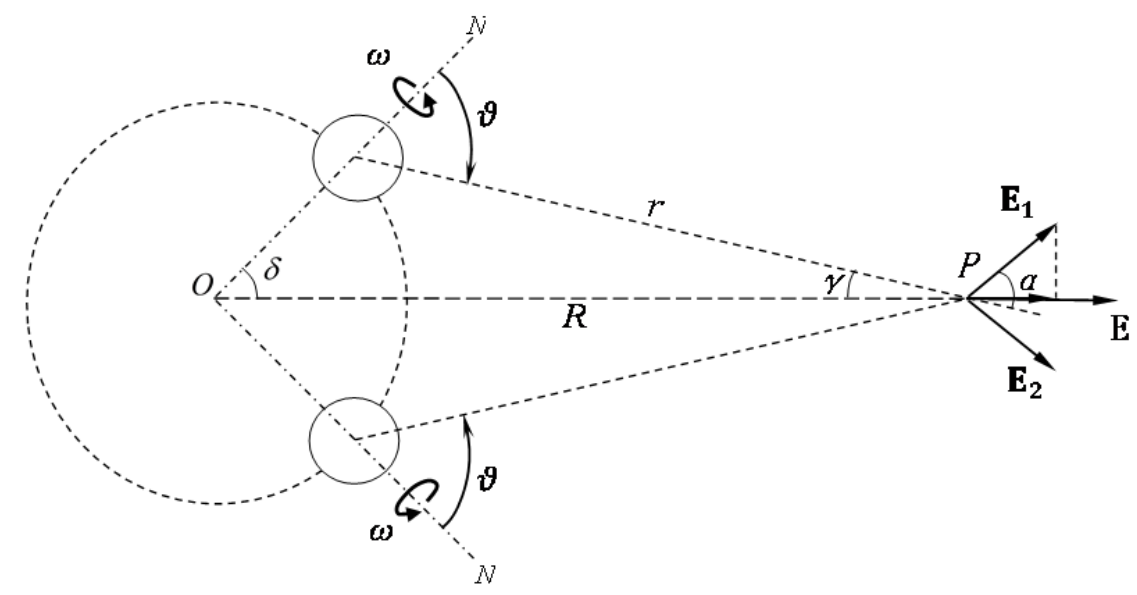

Figure 5. Resulting field of two symmetrically posed rotators

A proof of the geometric statement can be found in the appendix. From this statement it immediately follows that summarized electric vector $\mathbf{E}=\mathbf{E}_{1}+\mathbf{E}_{2}$ provided by two symmetric rotators (Figure 5) is always directed in parallel to the vector $\mathbf{O P}$ whatever values of an angle $\delta$ would be. It is obvious that if the direction of rotation both rotators is changed, the vector $\mathbf{E}$ will change to opposite directed one. Similarly, altering of the vector $\mathbf{E}$ would be due to the change the direction of magnetic axis of both rotators.

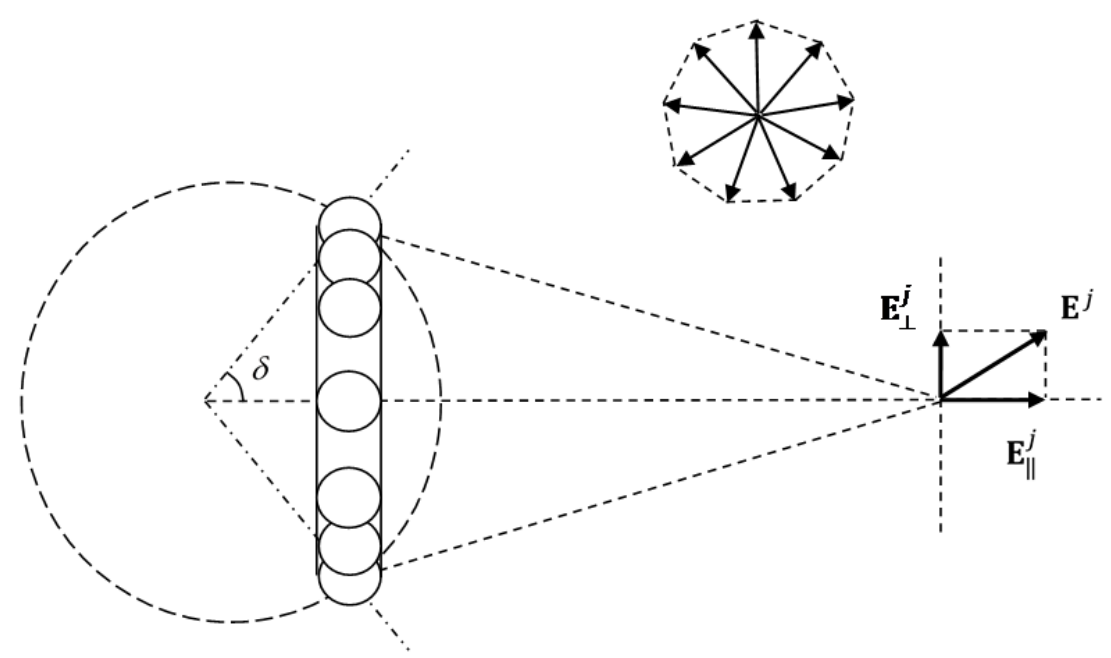

Figure 6. Resulting field of several symmetrically posed rotators

Let several rotators be placed on the sphere at the same angle $\delta$ (Figure 6). The electric vector $\mathbf{E}^{j}$ induced by any $j$-th rotator in the point $P$ may be resolved into a longitudinal component $\mathbf{E}_{\|}^{j}$ parallel to $O P$ and a transverse component $\mathbf{E}_{\perp}^{j}$ perpendicular to $O P$. In general, the longitudinal component of a vector $\mathbf{E}$ (the superscript $j$ may be dropped) is 


$$
\mathbf{E}_{\|}=\mathbf{E} \cos (C-\gamma)=\mathbf{E}(\cos \propto \cos \gamma+\sin \propto \sin \gamma)=\mathbf{E} \frac{\sin \vartheta \cos \gamma+2 \cos \vartheta \sin \gamma}{\sqrt{1+3 \cos ^{2} \vartheta}} .
$$

Here $\sin \propto$ and $\cos \propto$ are substituted through $\sin \vartheta$ and $\cos \vartheta$ from equations (3). This yields

$$
\mathbf{E}_{\|}=E \frac{\sin \vartheta \cos \gamma+2 \cos \vartheta \sin \gamma}{\sqrt{1+3 \cos ^{2} \vartheta}} \mathbf{u}_{\|}=\frac{\mu_{0} \omega m \sin \vartheta}{r^{2}}(\sin \vartheta \cos \gamma+2 \cos \vartheta \sin \gamma) \mathbf{u}_{\|}=E_{\|} \mathbf{u}_{\|},
$$

where $\mathbf{u}_{\|}$is the longitudinal unit vector and $E_{\|}$is the projection's coordinate (having sign).

The transverse component of a vector $\mathbf{E}$ is

$$
\begin{gathered}
\mathbf{E}_{\perp}=\mathbf{E} \sin (\propto-\gamma)=\mathbf{E}(\sin \propto \cos \gamma-\cos \propto \sin \gamma)= \\
E \frac{2 \cos \vartheta \cos \gamma-\sin \vartheta \sin \gamma}{\sqrt{1+3 \cos ^{2} \vartheta}} \mathbf{u}_{\perp}=\frac{\mu_{0} \omega m \sin \vartheta}{r^{2}}(2 \cos \vartheta \cos \gamma-\sin \vartheta \sin \gamma) \mathbf{u}_{\perp}=E_{\perp} \mathbf{u}_{\perp},
\end{gathered}
$$

where $E$ is taken from (2), $\mathbf{u}_{\perp}$ is the transverse unit vector. When the angle $\vartheta$ varies from zero to $\pi / 2$, the value $2 \cos \vartheta \cos \gamma$ monotonically decreases from two to zero whereas the value $\sin \vartheta \sin \gamma$ monotonically increases from zero to some positive magnitude. Consequently, the expression $2 \cos \vartheta \cos \gamma-\sin \vartheta \sin \gamma$ change sign and therefore the transverse component $\mathbf{E}_{\perp}$ must necessarily reverse its direction. As it can be seen, both components $\mathbf{E}_{\|}$and $\mathbf{E}_{\perp}$ are small when $\vartheta$ together with $\sin \vartheta$ are small. When $2 \cos \vartheta \cos \gamma-\sin \vartheta \sin \gamma=0$, the angle $\propto$ becomes equal to the angle $\gamma=\gamma_{0}$. So the component $\mathbf{E}_{\perp}$ reverses its sign at the angle

$$
\vartheta=\vartheta_{0}=\arccos \left(\sin \gamma_{0} / \sqrt{1+3 \cos ^{2} \gamma_{0}}\right),
$$

which is readily calculated from the equation (4). The value of the angle $\gamma_{0}$ depends on the geometric parameters $b$ and $R$ of the system under consideration and position of the observer.

The vector sum $\sum_{j} \mathbf{E}_{\perp}^{j}$ of all transverse components would be zero if the rotator's centres are set at all of the vertices of a regular polygon (Figure 6, up). Hence, the vector sum $\sum_{j} \mathbf{E}_{\|}^{j}$, and due to the geometric statement the summarized electric vector $\mathbf{E}=\sum_{j} \mathbf{E}^{j}$ is directed along the radial ray $O P$ in parallel (or counter-parallel) to the vector OP whatever values of an angle $\delta$ would be.

\section{Spherical System of Many Magnetic Rotators}

Let $N \quad a$-rotators be evenly allocated all over $b$-sphere with a nearly random distribution. Consider a tight allocation of $N$ rotators on the sphere. The 'tight' here does mean all $N$ rotators are tightly embedded so that $N$ is the maximum number (depending on values $b$ and $a$ ) of $a$-rotators which may just be inserted onto the sphere. Approximately taken. $N \approx 0.9069\left(4 \pi b^{2}\right) / \pi a^{2}=4 p b^{2} / a^{2}$ Here the number $p$ is the packing density factor which is equal to 0.9069 for a hexagonal lattice, and to 0.7854 for a square one. The set of centers of tightly embedded rotators is to be said ' $N$ - ensemble'. Let $\left\{a_{n}\right\}$ be an indefinitely decreasing sequence of radii, $\left\{N_{n}\right\}$ be a correspondent sequence of $N$ - ensembles, and $\left\{\left(\mathbf{E}_{\perp}\right)_{n}\right\}$ be a correspondent sequence of transverse components $\mathbf{E}_{\perp}$ at a given point of view $P$. The sequence $\left\{\left(\mathbf{E}_{\perp}\right)_{n}\right\}$ of transverse components converges to zero.

Let us slice the $b$-sphere with a sequence of vertical planes all being orthogonal to the beam $O P$ (Figure 7), so that the width of every ring-shaped strip on the sphere is not more than $3 a$. Denote $N_{i}$ the quantity of rotators occurring in some stripe $\left(N=\sum_{i} N_{i}\right)$. The vector-diagram of the correspondent transverse components $\mathbf{E}_{\perp}^{i}\left(i=1,2, \ldots, N_{i}\right)$ has some small deviations from a regular one (the additional arrows to the up-left). Let us distinguish $N$ vertices of a regular polygon on the middle line of this stripe. Using small translations we can pose centers of all $N_{i}$ rotators overall vertices. An illustrative diagram of all additional vectors and the final sum (the bold one to the up-right) is shown in the Figure 7.

It is intuitively clear that similar operation made on all the strips does diminish the total vector-sum of shifts. The greater the number $N$ (or, rather, $N_{n}$ ), the smaller is total shift i.e. the value of the vector $\left(\mathbf{E}_{\perp}\right)_{n}$. A more striking formal proof is as follows. Let the stochastic event be a result of a chance throwing of the point onto the $b$ - sphere with uniform probability distribution. The $a$-rotator with centre at this point gives the vector $\mathbf{E}_{\perp}$ that is therefore a rectangular distributed random variable. Its expectation is zero because of the spherical symmetry. Due to the law of large numbers the sum of $N$ similar random variables tends to zero when $N$ unlimitedly increases. The chance throwing of the point onto the finite set of $a_{n}$-rotators' centres -- $N_{n}$ ensemble -- gives a discretely distributed vector random variable $\mathbf{E}_{\perp}^{n}$. The sequence of discrete distributions converges to the continual rectangular one when $a_{n}$ infinitely decreases. Hence, the sequence $\left\{\left(\mathbf{E}_{\perp}\right)_{n}\right\}$ converges to zero. 


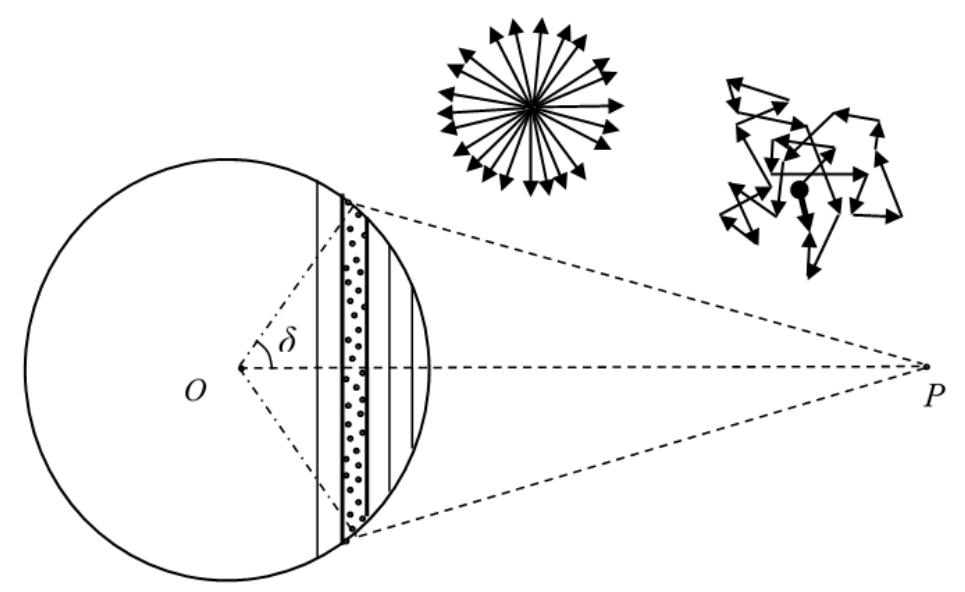

Figure 7. Stripe sections on the sphere

The field configuration is rather complicated in the near zone of the spherical system. Consider the field's behaviour in the far zone where the radius of the sphere is noticeably smaller then the length of the beam $O P$ $(b \ll R)$. Therefore, we may substitute respectively the values of $r, \sin \vartheta, \cos \vartheta$ for $R, \sin \delta, \cos \delta$ and the values of $\cos \gamma, \sin \gamma$ for 1 and $h / R=b \sin \delta / R$ in the expression (for single $a$-rotator)

$$
E_{\|}^{1}=\frac{\mu_{0} \omega m \sin \vartheta}{r^{2}}(\sin \vartheta \cos \gamma+2 \cos \vartheta \sin \gamma),
$$

and so obtain the new one

$$
E_{\|}^{1}=\frac{\mu_{0} \omega m}{R^{2}}\left(\sin ^{2} \delta+\frac{2 b}{R} \sin ^{2} \delta \cos \delta\right)
$$

that is correct to first order $(b / R)$ of magnitude (Figure 8).

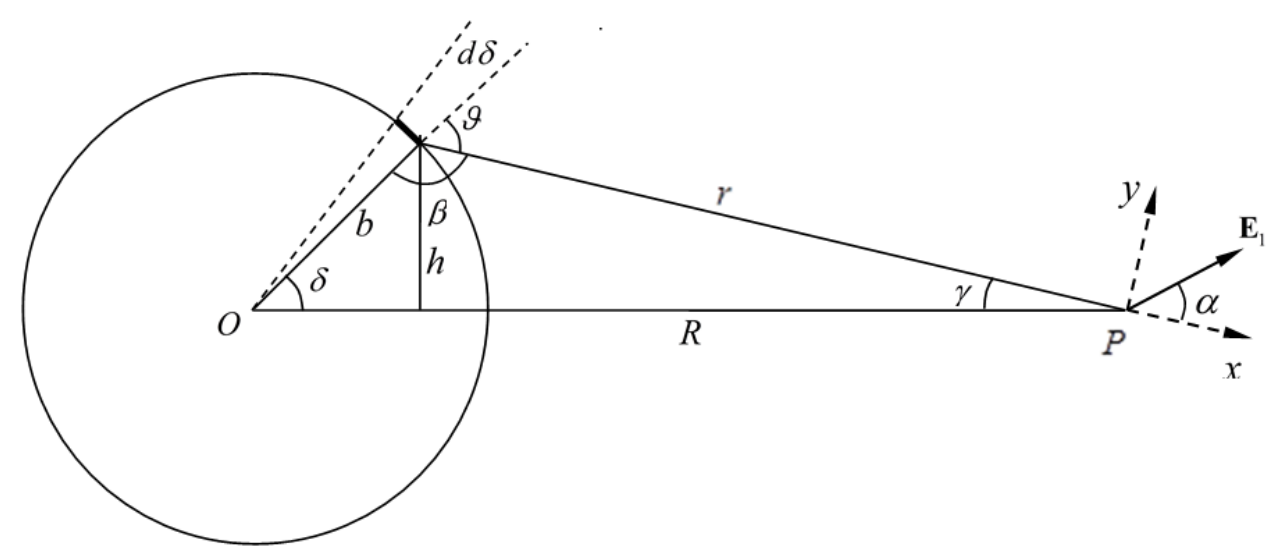

Figure 8. To the geometrical statement demonstration

The surface area of the $b$-sphere is $S=4 p b^{2}$. The surface area of a sliced strip between angles $\delta$ and $\delta+d \delta$ is $d S=2 \pi h b \cdot d \delta=2 \pi b^{2} \sin \delta \cdot d \delta$, and hence the quantity of $a$ - rotators on this strip is $d N=\left(\left(4 p b^{2} / a^{2}\right) / S\right) \cdot d S=\left(2 p b^{2} / a^{2}\right) \sin \delta \cdot d \delta$. The correspondent longitudinal coordinate of the vector $d \mathbf{E}$ is

$$
d E_{\|}=E_{\|}^{1} d N=\frac{2 p b^{2} \mu_{0} \omega m}{a^{2} R^{2}}\left(\sin ^{3} \delta+\frac{2 b}{R} \sin ^{3} \delta \cos \delta\right) d \delta .
$$


The total longitudinal coordinate would be $E=\int_{0}^{\pi} d E_{\|}(\delta)$ (here the sum $\sum_{i}$ is substituted for the integral, and the subscript "\|" at $E$ is omit since the transverse component $\mathbf{E}_{\perp}$ is stochastically negligible quantity). The compounding integrals would be

$$
\begin{gathered}
\int_{0}^{\pi} \sin ^{3} \delta d \delta=-\int_{0}^{\pi} \sin ^{2} \delta d \cos \delta=-\int_{0}^{\pi}\left(1-\cos ^{2} \delta\right) d \cos \delta=\left.\left(-\cos \delta+\frac{1}{3} \cos ^{3} \delta\right)\right|_{0} ^{\pi}=\frac{4}{3}, \\
\int_{0}^{\pi} \sin ^{3} \delta \cos \delta d \delta=\int_{0}^{\pi} \sin ^{3} \delta d \sin \delta=\left.\frac{1}{4} \sin ^{4} \delta\right|_{0} ^{\pi}=0 .
\end{gathered}
$$

The net result (using the expression for a dipole magnetic moment from the section 2) is

$$
E \approx \frac{2 p b^{2} \mu_{0} \omega m}{a^{2} R^{2}} \cdot \frac{4}{3}=\frac{2 p b^{2} \mu_{0} \omega \cdot 4 \pi a^{3} M}{a^{2} R^{2} \cdot 3} \cdot \frac{4}{3}=\frac{32}{9} \cdot \frac{\pi a p b^{2} \mu_{0} \omega M}{R^{2}}=\frac{q}{4 \pi \varepsilon_{0} R^{2}},
$$

where $\varepsilon_{0}$ is permittivity of vacuum. Thus a spherical system of many magnetic rotators provides (with an accuracy of order $b / R$ ) the Coulombian electric field, where

$$
q=\frac{16}{9} \cdot \pi p \cdot 2 a \cdot 4 \pi b^{2} \cdot \omega M \varepsilon_{0} \mu_{0}=\frac{16}{9} \cdot \pi p \cdot 2 a S \cdot \omega M / c^{2}
$$

is an effective (not actual!) charge, the value $(\mathrm{amp} \cdot \mathrm{sec}$ ) and the sign of which depend on the magnitudes and directions of vectors $\boldsymbol{\omega}$ and $\mathbf{M}$. Here $c$ is light speed in vacuum, $S$ is a surface area of the $b$-sphere, $2 a S$ is the volume of a spherical layer as thick as the diameter of $a$-rotator.

Examine now a magnetic field of the spherical system under consideration. Magnetic vector $\mathbf{B}_{1}$ of an $a$ rotator has two nonzero coordinates

$$
B_{r}^{1}=\frac{2 \mu_{0} m \cos \vartheta}{r^{3}}, \quad B_{\vartheta}^{1}=\frac{\mu_{0} m \sin \vartheta}{r^{3}} .
$$

The longitudinal component parallel (or antiparallel) to the vector $\mathbf{O P}$ has the coordinate

$$
B_{\|}^{1}=\left(\mathbf{B}_{1} \cdot \mathbf{u}_{\|}\right)=\frac{\mu_{0} m}{r^{3}}(2 \cos \vartheta \cos \gamma-\sin \vartheta \sin \gamma),
$$

where $\mathbf{u}_{\|}$is the unit vector of the radial ray $O P$ which $r$ - and $\vartheta$ - coordinates are $\cos \gamma$ and $(-\sin \gamma)$ respectively. Using the precedent substitution we get in the far zone

and hence

$$
B_{\|}^{1}=\frac{\mu_{0} m}{R^{3}}\left(2 \cos \delta-\frac{b}{R} \sin ^{2} \delta\right),
$$

$$
d B_{\|}=B_{\|}^{1} \cdot d N=B_{\|}^{1} \cdot \frac{N}{S} d S=\frac{2 p b^{2} \mu_{0} m}{a^{2} R^{3}}\left(2 \cos \delta \sin \delta-\frac{b}{R} \sin ^{3} \delta\right) .
$$

The coordinate of the total longitudinal component of magnetic vector would be $B=\int_{0}^{\pi} d B_{\|}(\delta)$, where the integral

$$
\int_{0}^{\pi} 2 \cos \delta \sin \delta d \delta=2 \int_{0}^{\pi} \sin \delta d \sin \delta=\left.\sin ^{2} \delta\right|_{0} ^{\pi}=0
$$

and the integral $\int_{0}^{\pi} \sin ^{3} \delta d \delta=4 / 3$, equation (5). Finally, $B=-\frac{8 p b^{3} \mu_{0} m}{3 a^{2} R^{4}}$, i.e. the rate of decrease for the magnetic field (with moving off the $b$-sphere) is two orders faster then that for the electric one. The transverse component $\mathbf{B}_{\perp}$ being stochastically negligible, the field of the spherical system of many magnetic rotators is asymptotically Coulombian. Thus we have a charge-free Coulomb-wise field.

\section{Spherical System of Many Electric Rotators}

The electro-kinematical effect "symmetric" to the Zajev-Dokuchajev one is known for a long time. The dragging of electric fields by its charge carrier rotating about an axis has been examined by H. Rowland (Thomson, 1893, p.23; Rowland, 1878) in the XIX century and by A. Eihenwald (Eihenwald, 1956)) at the very inception of the XX. Taking this effect into account, consider a spherical system similar to the one given above but combined with many electric $a$-rotators. 
An electric $a$-rotator represents a solid ball carrying the charge $q$ evenly distributed over the volume or over the spherical surface. In the external space we have Coulombian electric field quite like that providing by point charge $q$ located in the ball's centre: $\mathbf{E}=\frac{q}{4 \pi \varepsilon_{0} r^{2}} \mathbf{u}_{r}$. A unit vector $\mathbf{u}_{r}$ is directed outward from the centre to a point of view. This ball rotates in a vacuum about some diameter at a constant angle velocity $\omega$. Due to the electro-kinematical Rowland- Eihenwald effect at a given point with radius-vector $\mathbf{r}$ we have a magnetic vector $\mathbf{B}=1 / \mathrm{c}^{2} \mathbf{v} \times \mathbf{E}=\frac{q}{4 \pi \varepsilon_{0} c^{2} r^{2}} \mathbf{v} \times \boldsymbol{u}_{r}$, where $\mathbf{v}$ is the linear velocity corresponding to $\boldsymbol{\omega}$. The square of light speed in denominator here determines the smallness of the effect and hence the difficulties of measurement that have been completely surmounted only in the experiments by A. Eihenwald.

Let the axle of rotation of an electric $a$-rotator be the polar axis of a spherical coordinates $(r, \vartheta, \varphi)$. At a point of view the components of speed $\mathbf{v}$ are $v_{r}=0, v_{\vartheta}=0, v_{\varphi}=\omega r \sin \vartheta$. Then components of the magnetic vector B would be,

$$
\mathbf{B}_{r}=0, \quad \mathbf{B}_{\vartheta}=B_{\vartheta} \mathbf{u}_{\vartheta}=\frac{q \omega r \sin \vartheta}{4 \pi \varepsilon_{0} c^{2} r^{2}} \mathbf{u}_{\vartheta}, \quad \mathbf{B}_{\varphi}=0 .
$$

The spherical system under consideration is similar to the system which has been examined above, merely all magnetic $a$-rotators are substituted for electric $a$-rotators.

Let several electric $a$-rotators be placed on the $b$-sphere at the same angle $\delta$. The value $\sin \gamma$ may be presented as $\sin \gamma=(b \sin \delta) / r$ (Figure 8). The projection of the magnetic vector $\mathbf{B}_{1}$ (due to the electro-kinematical effect) at the radial ray $O P$ is

$$
B_{\|}^{1}=\left(\mathbf{B}_{1} \cdot \mathbf{u}_{\|}\right)=B_{\vartheta} \cdot(-\sin \gamma)=-B_{\vartheta} \frac{b \sin \delta}{r}=\frac{-b q \omega \sin \delta}{4 \pi \varepsilon_{0} c^{2} r^{2}} \sin \vartheta .
$$

In the far zone we have

$$
B_{\|}^{1}=\frac{-b q \omega \sin \delta}{4 \pi \varepsilon_{0} c^{2} R^{2}} \sin \delta, \quad d B_{\|}=B_{\|}^{1} \cdot \frac{N}{S} d S=-\frac{2 p b^{3} q \omega \sin ^{3} \delta}{4 a^{2} \pi \varepsilon_{0} c^{2} R^{2}} d \delta
$$

and $B_{\|}=\int_{0}^{\pi} d B_{\|}(\delta) d \delta$.

According to (5), the integral $\int_{0}^{\pi} \sin ^{3} \delta d \delta=4 / 3$, so the magnetic field in the far zone is

$$
B=\frac{-2 p b^{3} q \omega}{3 \pi a^{2} \varepsilon_{0} c^{2} R^{2}}=\frac{-2 p b^{3} \mu_{0} q \omega}{3 \pi a^{2} R^{2}} .
$$

The subscript "\|" at $B$ is omit since the transverse component $\mathbf{B}_{\perp}$ is statistically insignificant. The equation (6) is a description of the magnetic monopole field where magnetic induction varies according to the inverse square law. It is apparent that besides the magnetic field, the Coulombian electric field provided by the total charge $Q=N q$ is present. Thus we have a "steady mixture" of the tantamount magnetic and electric fields. Both proved to be of the same-rate evolution at a distance (despite the case of a charge-free Coulomb-wise field).

A pure magnetic monopole field (6) is also plausible, for instance, in the case when one half of all rotators consist of positively charged ones, whereas others are negatively charged. Furthermore, positive and negative rotators are located on the sphere in a chess-board manner (a staggered order) and twirl in opposite directions respectively. Thus we would have a negligible electric field of high degree multipole.

\section{Conclusion}

The very idea of a kinematic system having the spherical symmetry was presented to the local Siberian Conference (Leus, 1998, pp. 134-148). There was adduced a version of the spherical system of magnetic rotators only, without any mathematical substantiation. As it has been seen in the above text, the magneto-kinematical effect gives an electro-kinematical field whereas the electro-kinematical effect gives a magneto-kinematical field. The magneto-electric systems examined in the present paper restore a "genetic" equality between electricity and magnetism. This equality stems from the fundamental symmetry between Rowland-Eihenvald effect ( $\mathbf{B}=$ $\left.1 / \mathrm{c}^{2} \mathbf{v} \times \mathbf{E}\right)$ on the one hand and Zajev-Dokuchajev effect $(\mathbf{E}=\mathbf{B} \times \mathbf{v})$ on the other, provided that fields of kinematic origin may actually exist. How could this statement be questioned? "Equation $\operatorname{div} \mathbf{B}=0$ (4.19) is 
one of the fundamental laws of electromagnetism, on the same footing as Coulomb's law. There have been no observed violations of this law that the divergence of the magnetic field $\mathbf{B}$ is everywhere zero; no free magnetic 'charges' have yet been discovered. The development of the theory of electromagnetism depends on this last negative observation. Since electromagnetic theory always gives the right answer we have very strong indirect evidence for the general validity of the Equation (4.19)" (Grant \& Phillips, 1998, pp.126-127). The properties of the examined spherical systems are not in an irreconcilable contradiction with this "one of the fundamental laws of electromagnetism". A point of the conciliation is abiding in the fact that despite non-zero divergence of the magneto-kinematical field, provided by the spherical system of electric rotators, no free magnetic charges appeared out.

Some important experimentation has yet to come. Although theoretically speaking linear equations meet the superposition principle, I should like to buck some physicists up for a scientific checking: the validity of the superposition for kinematical fields ought to be proven experimentally. For this purpose it isn't necessary to build a spherical system including thousands of elements. Two rotators only would be enough to verify this because the superposition is a transitive property. Should such an appropriate test prove to be positive, the spherical magneto-electric system will be something that is theoretically possible but has not been made yet. Nonetheless, who could warrant us that the Nature did not make this at the sub-microscopic level? "It is doubt that compels us into investigation, and by investigating that we recognize the truth" /P. Abelard (1079 -- 1142)/. This is an endless upward spiral of cognition.

As it has been underlined in the section 1, "electricity of kinematical origin does exist in the laboratory's non-rotating frame". Nonetheless, it seems fair at this point to raise a question about a specific attribute of each rotatory movement, namely acceleration. Let us refer to an estimate made by a Russian physicist and Nobel prize winner I.E. Tamm. "The impact of acceleration, i.e. force of inertia, on electrons in an accelerated media is equivalent to presence of some effective electric field with strength

$$
\mathbf{E}^{\prime}=-\frac{m}{e}\left(\frac{\partial \mathbf{u}}{\partial t}+(\mathbf{u} \nabla) \mathbf{u}\right)
$$

For example, consider a disk of radius a rotating with an angular velocity $\omega$. The maximal value of effective electric strength would be $E^{\prime}=a \omega^{2}(\mathrm{~m} / e)$. Taking $a=5 \mathrm{~cm}, \omega=100 \pi / \mathrm{sec}$ and $\mathrm{m} / e=1.9 \cdot 10^{-19}$, we get $E^{\prime} \approx 3 \cdot 10^{-10}$ volt. Needless to say, that is quite trifling" (Tamm, pp. 538-539). In addition let us refer to the above cited book by Panofsky and Phillips: "It should be emphasized, however, that within the framework of special relativity our earlier considerations do permit us to describe all fields in all Lorentz frames, whether the sources are accelerated or not" (Panofsky \& Phillips, 1975, p. 339).

\section{Appendix}

This appendix contains the detailed proof of the geometrical statement.

In the spherical coordinates of the upper (first) rotator (Figure 5) we have the above defined angle $\propto$, and angle $\beta$ which is complementary to the $\vartheta$ (Figure 8).

Let $\mathbf{u}_{1}$ be a unit vector for the direction of vector $\mathbf{E}_{1}$ and $\mathbf{u}_{O P}$ be a unit vector for the direction of the radial ray $O P$. The Cartesian coordinates $(x, y)$ of the vector $\mathbf{u}_{1}$ are $\cos \propto, \pm \sin \propto$ (Figure 8). The up sign relates to the front side of spherical system whereas the down sign relates to the rear one according to the Bogach's formula (1) for $E_{\vartheta}$. The Cartesian coordinates of the vector $\mathbf{u}_{O P}$ are $\cos \gamma, \sin \gamma$. Then using the formulae (3) for the inner product $\mathbf{u}_{1} \cdot \mathbf{u}_{O P}$ we can write:

$$
\mathbf{u}_{1} \cdot \mathbf{u}_{O P}=\cos \left(\mathbf{E}_{1}, \mathbf{O P}\right)=(\cos \propto \cos \gamma \pm \sin \propto \sin \gamma)=\frac{\sin \vartheta \cos \gamma \pm 2 \cos \vartheta \sin \gamma}{\sqrt{1+3 \cos ^{2} \vartheta}}=\frac{f}{\sqrt{1+3 \cos ^{2} \vartheta}} .
$$

Now let us put attention on the numerator, denoted by $f$. Substitution the angle $\vartheta$ for the complementary angle $\beta$ (Figure 8 ) in this expression gives

$$
f=\sin \vartheta \cos \gamma \pm 2 \cos \vartheta \sin \gamma=\sin \beta \cos \gamma \mp 2 \cos \beta \sin \gamma .
$$

Using the formulae of elementary trigonometry provides us the following:

$$
\sin \gamma=\frac{h}{r}, \sin \beta=\frac{R}{b} \sin \gamma=\frac{R h}{b r}, \cos \gamma=\frac{R^{2}+r^{2}-b^{2}}{2 R r}, \cos \beta=\frac{b^{2}+r^{2}-R^{2}}{2 b r} .
$$

Substituting the above expressions in $f$, it is obtained: 


$$
f(r)=\frac{R h}{b r} \cdot \frac{R^{2}+r^{2}-b^{2}}{2 R r} \pm 2 \cdot \frac{R^{2}-r^{2}-b^{2}}{2 b r} \cdot \frac{h}{r}=\frac{h}{2 b r^{2}}\left(R^{2}+r^{2}-b^{2} \mp 2 R^{2} \pm 2 b^{2} \pm 2 r^{2}\right) .
$$

Now we separate two cases:

$$
\begin{aligned}
& g_{1}(r)=R^{2}+r^{2}-b^{2}-2 R^{2}+2 b^{2}+2 r^{2}=3 r^{2}+b^{2}-R^{2} ; \\
& g_{2}(r)=R^{2}+r^{2}-b^{2}+2 R^{2}-2 b^{2}-2 r^{2}=3 R^{2}-3 b^{2}-r^{2} .
\end{aligned}
$$

Let us examine the behaviour of these functions in the ranges $R-b \leq r \leq \sqrt{R^{2}-b^{2}}$ and $\sqrt{R^{2}-b^{2}} \leq r \leq$ $R+b$ (front part of the $b$-sphere and rear part of the $b$-sphere, respectively) of the variable $r$, which can vary between limits: $R-b \leq r \leq R+b$. We have

$$
g_{1}(r) \geq 3(R-b)^{2}+b^{2}-R^{2}=3 R^{2}-6 R b+3 b^{2}+b^{2}-R^{2}=2\left(R^{2}-3 R b+2 b^{2}\right)=2(R-b)(R-2 b) .
$$

And similarly

$$
g_{2}(r) \geq 3 R^{2}-3 b^{2}-(R+b)^{2}=(R+b)(3 R-3 b-R-b)=(R+b)(2 R-4 b)=2(R+b)(R-2 b)
$$

It is evident that $g_{1}(r)>0$ and $g_{2}(r)>0$, if $R>2 b$. So, in the space at any point $P$, where the condition $R>2 b$ is fulfilled, the function $f(r)$ is positive for all values of the variable $r$, when the positioning angle $\delta$ varies between 0 and $\pi: R-b \leq r \leq R+b$. Therefore by virtue of (7), the value $\cos \left(\mathbf{E}_{1}, \mathbf{O P}\right)$ is positive, i.e. the orthogonal projection of the vector $\mathbf{E}_{1}$ at the radial ray $O P$ does not change its sign. The geometrical statement is proven.

The corollary.

It is obvious that inside the spherical system, where the total surface of the $b$-sphere is a "rear side", the function $g_{2}(r)=3 R^{2}-3 b^{2}-r^{2}$ only is valid. Let us examine the behaviour of the function $g_{2}(r)$ when $R<b$. For $r=b$ we have $g_{2}(r)=3 R^{2}-4 b^{2}<0$. The roots $r_{1,2}= \pm \sqrt{3\left(R^{2}-b^{2}\right)}$ of the equation $g_{2}(r)=0$ both are a conjugate complex number. Hence the function $g_{2}(r)$ is negative for all $r$ from the segment $[b-R, b+R]$. Consequently, the radial direction of the induced electric field inside the $b$-sphere is in opposition to the one outside the sphere of radius $2 b$. Between these two boundaries $(b<R<2 b)$ a transient zone is disposed.

\section{Acknowledgement}

The author sincerely thanks Mr Michael Kyle for the proof-reading of this text.

\section{References}

Bogach, V. A. (1996). A hypothesis about existence of a static electromagnetic field and its properties (Preprint 13-96-463). Dubna, Russia: JINR (in Russian).

Eihenwald, A. A. (1956). Izbrannye raboty (Selected works). Moscow: Physico-matematicheskaja Literatura. (in Russian)

Grant, I. S., \& Phillips, W. R. (1998). Electromagnetism (2nd ed.). Chichester: John Wiley \& Sons.

Jackson, J. D. (1975). Classical Electrodynamics (2nd ed.). New York: John Wiley \& Sons.

Leus, V. A. (1998). Some pending issues in the theoretical base of electrodynamics. Proceedings of the 2nd Siberian Conference on Space-time Physics (pp. 134-148). Novosibirsk: Institute of Mathematics Press (in Russian).

Leus, V. A. (2013). Magneto-kinematical and Electro-kinematical Fields. Progress in Electromagnetics Research-M., 32, 27-41.

Leus, V. A. (2014). On the Field of a Moving Charge. Open Journal of Modern Physics, 1(1), 1-7. Retrieved from http://scipublish.com/journals/MPHY/papers/84

Leus, V. A., \& Smith, B. (2014). On a New Source of Electromagnetic Radiation: Experimental Evidence. Applied Physics Research, 6(3), 127-138. http://dx.doi.org/10.5539/apr.v6n3p127

Leus, V. A., \& Zatolokin, V. N. (2006). Magnetokinematical effect. International Journal of Electrical Engineering Education (IJEEE), 43(3), 245-251.

Leus, V. A., Smith, R. T., \& Maher, S. (2013a). The Physical Entity of Vector Potential in Electromagnetism. Applied Physics Research, 5(4), 56-68. http://dx.doi.org/10.5539/apr.v5n4p56 
Leus, V. A., Smith, R. T., \& Maher, S. (2013b). Two Types of Electromagnetic Induction Existing in Nature. PIER Symposium. Stockholm Sweden, August, 12-15.

Leus, V., \& Taylor, S. (2011). On the motion of the field of a permanent magnet. Eur. J. Phys., 32(5), 1179-1192. http://dx.doi.org/10.1088/0143-0807/32/5/006

Leus, V., \& Taylor, S. (2012). Experimental Evidence for the Magneto-kinematic Effect. PIERS Proceedings (pp. 1040-1048). Moscow, Russia, August 19-23.

Panofsky, W., \& Phillips, M. (1975). Classical Electricity and Magnetism. Cambridge: Addison - Willey.

Pohl, R. W. (1960). Electrizitätslehre. Berlin: Springer.

Rajaraman, K. C. (2008). The field of rotating cylindrical magnet. International Journal of Electrical Engineering Education (IJEEE), 45(1), 34-45.

Rowland, H. (1878). On the Magnetic Effect of Electric Convection. American Journal of Science, XV(3), 30-33.

Schiff, L. I. (1939). A Question in General Relativity. Proc. Nat. Acad. Sci. (USA), 25, 391-395.

Tamm, I. E. (1976). Osnovy Teorii Electrichestva - Fundamentals of the Electricity Theory (9th ed.). Moscow: Nauka (in Russian).

Taylor, S., \& Leus, V. (2012). The magneto-kinematic effect for the case of rectilinear motion. Eur. J. Phys., 33(4), 837-852. http://dx.doi.org/10.1088/0143-0807/33/4/837

Thomson, J. J. (1893). Notes on recent research in electricity and magnetism. Oxford: Clarendon Press.

Weisstein, E. W. (1999). Concise Encyclopaedia of Mathematics. London: CRC Press.

\section{Copyrights}

Copyright for this article is retained by the author(s), with first publication rights granted to the journal.

This is an open-access article distributed under the terms and conditions of the Creative Commons Attribution license (http://creativecommons.org/licenses/by/3.0/). 